Proceedings of the 42th "Jaszowiec" International School and Conference on the Physics of Semiconductors, Wisła 2013

\title{
Properties of Excitons in Quantum Dots with a Weak Confinement
}

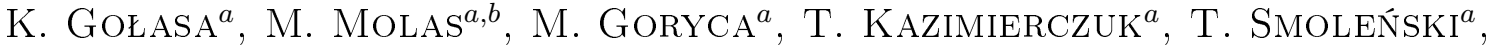

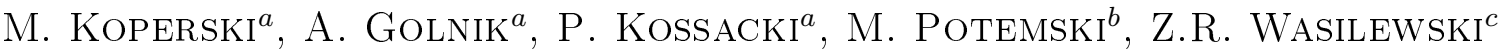 \\ AND A. BABIŃSKI ${ }^{a}$ \\ ${ }^{a}$ Institute of Experimental Physics, Faculty of Physics, University of Warsaw, Hoża 69, 00-681 Warsaw, Poland \\ ${ }^{b}$ Laboratoire National des Champs Magnetiques Intenses, Grenoble, France \\ ${ }^{c}$ Institute for Microstructural Sciences, NRC Canada, Ottawa, Canada
}

\begin{abstract}
Statistical properties of neutral excitons, biexcitons and trions confined to natural quantum dots formed in the InAs/GaAs wetting layer are reported. The correlation of the trion binding energy and the biexciton binding energy was found. Magnetospectroscopic measurements of the excitons revealed also the correlation of excitonic effective $g^{*}$ factor of an exciton with the biexciton binding energy. The qualitative picture of the effect of quantum confinement on the observed correlations is presented.
\end{abstract}

DOI: 10.12693 /APhysPolA.124.781

PACS: 78.55.Cr, 78.67.Hc

\section{Introduction}

Quantum confinement of carriers in semiconductor quantum dots (QDs) leads to numerous effects of fundamental character. This makes them objects of intense study (for a review, see Ref. [1]). The research is driven by both scientific curiosity and promising optoelectronic applications, e.g., in optical quantum devices based on single-photon emission. One of the problems, hindering their use in such applications is the statistical character of the QDs potential structure which leads to inhomogeneous broadening of their emission energies. The energy structure of carrier complexes confined to QDs depends on the confining potential and electrostatic interactions between the carriers. Substantial efforts have been made to relate the morphological properties to the properties of excitons confined in QDs [2]. The key point lies in the identification of main factors, which affect the excitonic structure of QDs. To this goal we studied optical properties of excitons confined in natural dots formed in the InAs/GaAs wetting layer (WL) [3, 4]. The three-dimensional quantum confinement of carriers in the WL is related to potential fluctuations due its In-composition fluctuations [5]. Deepest potential fluctuations resulted from the WL disorder confine both electrons and holes and at low temperature their recombination results in the optical emission at the energy up to $10-15 \mathrm{meV}$ below the threshold of the WL-related emission. The fluctuations are referred to as natural InAs/GaAs quantum dots (WLQDs). The non-classical character of the optical emission related to excitons confined in the WLQDs was confirmed by the observation of biexciton-exciton emission cascades in the WLQDs [6] which makes the WLQDs a potential system to be used in quantum information processing applications.

The sample investigated in this work was grown by molecular beam epitaxy using the In-flush technique.
It contained a single layer of self-assembled $\mathrm{InAs} / \mathrm{GaAs}$ QDs grown at $524^{\circ} \mathrm{C}$, deposited on a GaAs substrate covered by an $800 \mathrm{~nm}$ GaAs buffer layer. The sample was capped with a $100 \mathrm{~nm}$ GaAs top layer. The indium flush [7] was applied to the self-assembled QDs at $5 \mathrm{~nm}$, resulting in formation of flat dots of $3.5 \mathrm{~nm}$ height. Low temperature micro-photoluminescence ( $\mu$-PL) measurements were performed using continuous-flow cryostat or in a bath-type cryostat. In the latter case the high spatial resolution was assured in the experiment by using a specially designed reflection-type microscope objective immersed together with the sample inside the cryostat. The diameter of the laser spot on the sample surface was lower than $1 \mu \mathrm{m}$. Two different lasers were employed to excite the sample: nonresonant CWNd:YAG $(532 \mathrm{~nm})$ and LED laser $(650 \mathrm{~nm})$. Measurements in high magnetic fields were performed in an optical fiber-based experimental setup in the National High Magnetic Field Laboratory in Grenoble with attocube piezoelectric positioners allowing for a precise positioning of the sample [8]. The photoluminescence was resolved using a $0.5 \mathrm{~m}$ diffraction spectrometer with a CCD camera.

\section{Experimental results}

Basic properties of the WLQDs-related emission lines observed in $\mu$-PL measurements at low temperature can be summarized as follows.

At low excitation power density, the spectrum usually consists of two emission lines: $\mathrm{X}$ and $\mathrm{X}^{+}$(see Fig. 1). Similar $\mu$-PL spectral lineshape can be found on several spots of the sample. The inspection of the CCD camera image confirms that both lines originate from the same location. The WLQDs-related emission is spatially anti-correlated with emission from self-assembled QDs present in the structure (which are not addressed in this 
work). The average areal density is of the order of $10^{7}$ to $10^{8} \mathrm{~cm}^{-2}$. Polarization-sensitive measurements confirm that the $\mathrm{X}$ emission line is split into two components polarized in perpendicular directions. The splitting is due to the long-range component of an anisotropic electronhole exchange interaction [9] and supports the attribution of the $\mathrm{X}$ line to a neutral exciton.

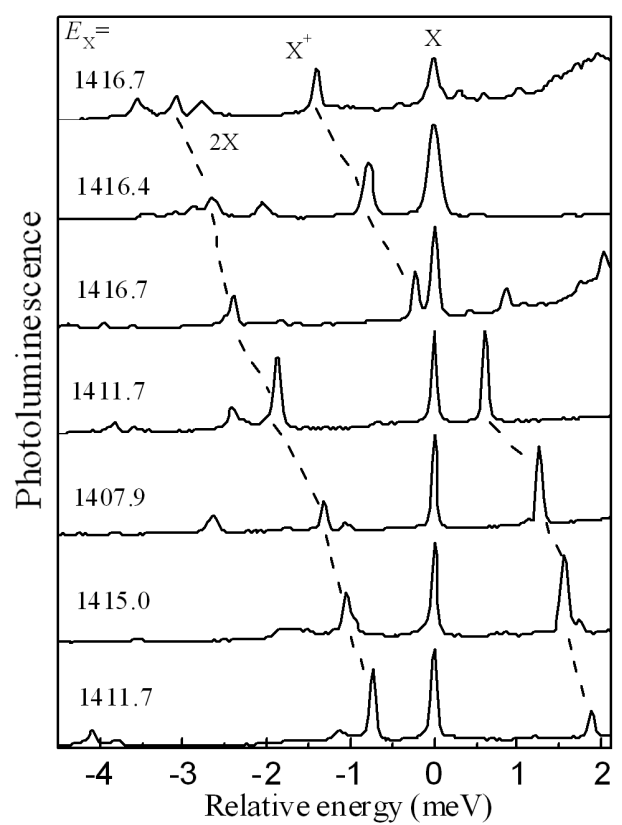

Fig. 1. Photoluminescence spectra of several WLQDs with an exciton $(\mathrm{X})$, biexciton $(2 \mathrm{X})$ and a positive trion $\left(\mathrm{X}^{+}\right)$emission lines. Note energy scale relative to the exciton emission energy. Measurements are taken at $T=1.6 \mathrm{~K}$

The energy splitting changed from dot to dot and it was usually less than $20 \mu \mathrm{eV}$. No $\mathrm{X}^{+}$splitting is observed, which is consistent with its attribution to a trion, in which the exchange interaction is quenched. The attribution of the trion to the positively charged exciton is based on background acceptor presence in the intentionally undoped GaAs layers [10]. Also its usual appearance in the spectrum is consistent with the larger probability for holes to be localized by potential fluctuations due to their higher mass as compared with the mass of electrons [11]. Depending on excitation conditions [6] another emission line related to a trion was observed in the spectrum, which is related to a negatively charged exciton $\mathrm{X}^{-}$.

Both $\mathrm{X}$ and $\mathrm{X}^{+}$emission lines gain in intensity with increasing excitation power. With the further increase of the excitation power, another emission line $2 \mathrm{X}$ emerges in the spectrum with increasing excitation power at the energy $E_{2 \mathrm{x}}$, which is lower than the neutral exciton emission energy $E_{\mathrm{X}}$. The $2 \mathrm{X}$ emission line dominates the spectrum excited with the highest power density. The $2 \mathrm{X}$ consisted of two components, which were linearly polarized in perpendicular directions and its splitting was opposite to the neutral exciton splitting. The observation confirms our attribution of the $2 \mathrm{X}$ to the recombination of a neutral biexciton.

A set of spectra related to several WLQDs are presented in Fig. 1 (note the energy scale relative to the energy of a neutral exciton $E_{\mathrm{X}}$ ). It can be seen that the positive trion binding energy $E_{\mathrm{X}+}^{\mathrm{B}}=E_{\mathrm{X}+}-E_{\mathrm{X}}$ correlates with the biexciton binding energy $E_{2 \mathrm{X}}^{\mathrm{B}}=E_{2 \mathrm{X}}-E_{\mathrm{X}}$. Moreover with the decreasing biexciton binding energy, the positive trion binding energy becomes positive (the trion is said to become unbound). In order to gain more insight into the properties of excitons in WLQDs magneto-spectroscopic studies were also performed.

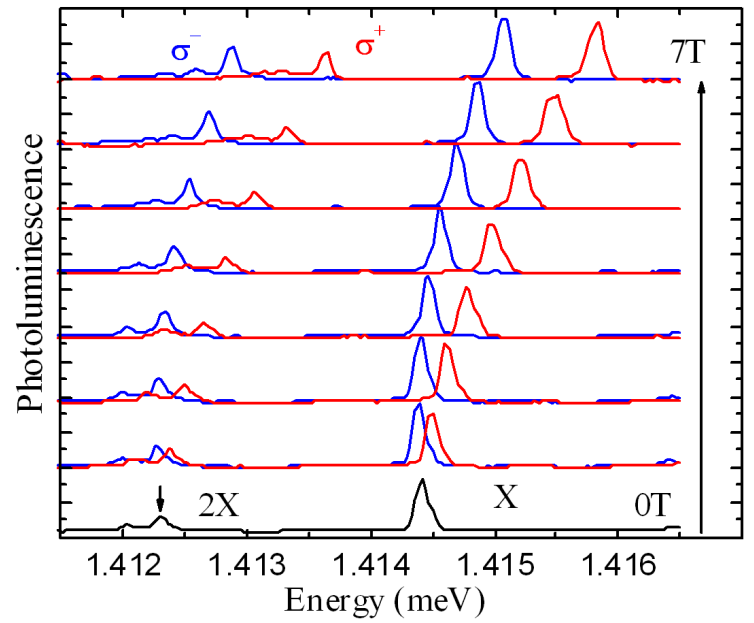

Fig. 2. Polarization-sensitive photoluminescence spectra from a single WLQD in magnetic field.

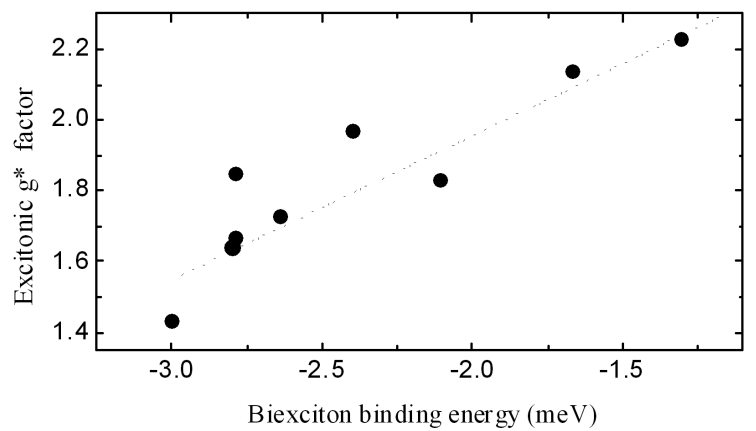

Fig. 3. The effective $g^{*}$-factor of neutral excitons confined to the WLQDs as a function of the biexciton binding energy. The line is the guide to the eye.

Typical results of polarization-resolved measurements done in the Faraday configuration are presented in Fig. 2. It was observed that the excitonic emission lines split in magnetic field into two circularly polarized components and shift their emission to higher energy [12]. In moderate magnetic fields the field dispersion of the $\mathrm{X}$ excitonic emission can be expressed as 


$$
E(B)=E(0) \pm \frac{1}{2} g^{*} \mu_{\mathrm{B}} B+\gamma B^{2},
$$

with the Bohr magneton, $\mu_{\mathrm{B}}=5.79 \times 10^{-5} \mathrm{eV} / \mathrm{T}$. The second term in the expression corresponds to the energy splitting between two circularly polarized components of the bright exciton $(|M|=1)$ of opposite momentum which is due to the Zeeman interaction of the spin with external magnetic field. The interaction is characterized by the effective excitonic $g^{*}$-factor. The diamagnetic shift of excitonic emission, which can be parameterized with a diamagnetic coefficient $\gamma$ will not be addressed in this communication. The effective excitonic $g^{*}$ of neutral excitons measured for several WLQDs are collected in Fig. 3. As it can be seen in the figure, the excitonic $g^{*}$ also correlates with the biexciton binding energy.

\section{Discussion}

The remarkable correlation between the positive trion binding energy and the biexciton binding energy observed in the investigated structure suggests that despite fluctuations in the WLQD morphology some fundamental rules hold for the properties of confined excitons. Similar behavior was previously observed in self-assembled InAs/GaAs QDs [13], CdTe/ZnTe QDs [14] and strain-free GaAs/GaAlAs QDs [10]. While in the InAs/GaAs and $\mathrm{CdTe} / \mathrm{ZnTe} \mathrm{QDs}$ the positive trion follows the biexciton in a relatively narrow energy range, in the strain free GaAs/GaAlAs QDs the correlation is similar to the observed in our experiment. In particular the transition of the positive trion from the bound to the unbound was observed. The correlation was interpreted in terms of the balance between the Hartree mean-field corrections to the single-particle states and the inter-particle correlations [10].

Some conclusions on the properties of excitons in the WLQDs can also be drawn from magnetospectroscopic data. Within the experimental accuracy, the excitonic emission is circularly polarized except for the smallest magnetic field. This confirms that the excitons are mainly composed of electrons and heavy holes. The composition of excitons results from the substantial splitting between heavy and light-hole subbands, which is related to substantial strain accommodated in the WL. Despite the splitting, the mixing of the heavy with light-hole states cannot be neglected as two bright exciton states are split by the anisotropic exchange, which results in the linear polarization of their emission in zero magnetic field [15].

The $g^{*}$ factors in III-V QDs may have a rich structure resulting from strain, geometry, and confinement which makes the picture more complex than in the respective quantum wells (QWs). We believe however that our results can be qualitatively explained using the arguments previously proposed to explain the effect of quantum confinement on the $g^{*}$ factors in (In, Ga)As/GaAs QWs [16]. It is well established from $k \cdot p$ theory that the $g$ factors of carriers are determined by band-mixing effects. In particular a strong In-concentration and well-width dependence of the Zeeman splitting of heavy-hole excitons observed in the QWs was attributed to the effect of the coupling of heavy-hole and light-hole valence bands. The interaction affects the spin-up state of a heavy hole and reduces the heavy hole Zeeman splitting. The splitting between heavy holes and light-holes increases with both indium concentration and well width leading to the increase of the $g^{*}$ factor in the (In, Ga)As/GaAs QWs. Relatively large linear range of the Zeeman splitting in the investigated dots confirms that the splitting between heavy-holes and light-holes is larger than in the QWs investigated in Ref. [16]. In our opinion however its effect is sufficient to modulate the excitonic $g^{*}$ factors across the set of investigated dots. Therefore the monotonic dispersion of the effective $g^{*}$ factors with the biexciton binding energy reflects the magnitude of the heavy-hole light-hole mixing in the investigated dots.

In order to explain the observed behavior we propose a simplified picture of the effect of quantum confinement on the excitons in the WLQDs. The small biexciton binding energy corresponds to the largest $g^{*}$ factors. This must reflect large heavy-hole light-hole splitting due to a relatively high In-content of the dot. Deeper potential better confines electrons and holes and maximizes their overlap. The confinement evolves towards the strong confinement regime. In that case biexcitonic binding energy is small, as the repulsion between the same carriers is very similar to the attraction of carriers of opposite charge. Moreover as the spatial extent of the hole wavefunction is smaller than the extent of the electron, the binding energy of a positive trion is positive - the trion is said to be unbound. In the opposite limit, the carrier confinement is weaker which results in larger heavy hole-light-hole mixing and in smaller effective $g^{*}$ factor. The effect of the Coulomb interactions is more pronounced and it must be responsible for the larger biexcitonic binding energy and the negative binding energy of the positive trion.

\section{Conclusion}

We investigated optical properties of a set of the WLQDs. We have shown the characteristic pattern of excitonic emission spectra with a positive trion binding energy being proportional to the biexciton binding energy. Similar correlation of the excitonic $g^{*}$ factor with biexciton binding energy has been observed. We conclude that the binding energy of the biexciton carries non-trivial information on the properties of their confining potential in the investigated dots.

\section{Acknowledgments}

The work has been supported in part by the EuroMagNET II consortium under the EU contract no. 228043, by Polish Funds for Science 2009-2012, and the Foundation for Polish Science International PhD Projects Programme - co-financed by the European Regional Development Fund. 


\section{References}

[1] For a recent review of QDs properties see Handbook of Self-Assembled Semiconductor Nanostructures for Novel Devices in Photonics and Electronics, Ed. M. Henini, Elsevier, Amsterdam 2008.

[2] J.-W. Luo, A. Zunger, Phys. Rev. B 84, 235317 (2011).

[3] A. Babinski, J. Borysiuk, S. Kret, M. Czyz, A. Golnik, S. Raymond, Z.R. Wasilewski, Appl. Phys. Lett. 92, 171104 (2008).

[4] A. Babinski, M. Czyz, J. Borysiuk, S. Kret, A. Golnik, S. Raymond, J. Lapointe, Z.R. Wasilewski, Acta Phys. Pol. A 114, 1055 (2008).

[5] M. Hugues, M. Teisseire, J.-M. Chauveau, B. Vinter, B. Damilano, J.-Y. Duboz, J. Massies, Phys. Rev. B 76, 075335 (2007).

[6] T. Kazimierczuk, A. Golnik, P. Kossacki, J.A. Gaj, Z.R. Wasilewski, A. Babinski, Phys. Rev. B 84, 115325 (2011).

[7] Z.R. Wasilewski, S. Fafard, J.P. McCaffrey, J. Cryst. Growth 201-202, 1131 (1999).

[8] A. Babinski, S. Awirothananon, J. Lapointe, Z. Wasilewski, S. Raymond, M. Potemski, Physica E 26, 190 (2005).
[9] D. Gammon, E.S. Snow, B.V. Shanabrook, D.S. Katzer, D. Park, Phys. Rev. Lett. $\mathbf{7 6}$, 3005 (1996).

[10] M. Abbarchi, T. Kuroda, T. Mano, K. Sakoda, C.A. Mastrandrea, A. Vinatteri, M. Gurioli, T. Tsuchiya, Phys. Rev. B 82, 201301 (2010).

[11] E.S. Moskalenko, M. Larsson, W.V. Schenfeld, P.M. Petroff, P.O. Holtz, Phys. Rev. B 73, 155336 (2006).

[12] A. Babinski, A. Golnik, J. Borysiuk, S. Kret, P. Kossacki, J.A. Gaj, S. Raymond, M. Potemski, Z. Wasilewski, Phys. Status Solidi B 246, 850 (2009).

[13] M.-F. Tsai, H. Lin, C.-H. Lin, S.-D. Lin, S.-Y. Wang, M.-. Lo, S.-J. Cheng, M.-C. Lee, W.-H. Chang, Phys. Rev. Lett. 101, 267402 (2008).

[14] T. Kazimierczuk, T. Smolenski, M. Goryca, Ł. Kłopotowski, P. Wojnar, K. Fronc, A. Golnik, M. Nawrocki, J.A. Gaj, P. Kossacki, Phys. Rev. B 84, 165319 (2011).

[15] L. Besombes, K. Kheng, D. Martrou, Phys. Rev. Lett. 85, 425 (2000).

[16] N. Traynor, R.T. Harley, R.J. Warburton, Phys. Rev. B 51,7361 (1995). 